Théologiques

Théologiques

\title{
Les croyances et le croire chez des spiritualistes
}

\section{Deirdre Meintel}

Volume 13, numéro 1, printemps 2005

Croire et croyances

URI : https://id.erudit.org/iderudit/012529ar

DOI : https://doi.org/10.7202/012529ar

Aller au sommaire du numéro

\section{Éditeur(s)}

Faculté de théologie et de sciences des religions, Université de Montréal

\section{ISSN}

1188-7109 (imprimé)

1492-1413 (numérique)

Découvrir la revue

\section{Citer cet article}

Meintel, D. (2005). Les croyances et le croire chez des spiritualistes.

Théologiques, 13(1), 129-156. https://doi.org/10.7202/012529ar

\section{Résumé de l'article}

À première vue, l'objet de notre enquête, soit les croyances des spiritualistes, peut sembler très flou. Nous présenterons tout d'abord une topologie heuristique des croyances de spiritualistes membres du groupe sur lequel est centrée notre enquête. Le spiritualisme se distingue en effet par certaines croyances - on peut contacter les esprits des défunts et ces derniers peuvent venir en aide aux vivants - ainsi que par sa conception des dons spirituels dont chaque être humain serait doté. La guérison par les mains ou à distance, la clairvoyance, la clairaudience et la psychométrie (voyance à travers les objets) figurent ainsi parmi les dons possibles. Puis, nous essaierons de montrer que le caractère apparemment flou des croyances (la diversité de leur provenance, la variabilité des croyances au sein du groupe spiritualiste) n'empêche pas que la croyance religieuse s'épanouisse dans la vie de ces gens. Nous examinerons à cet effet de quelle manière les pratiques spécifiques au spiritualisme (par comparaison aux religions classiques en Occident), soit la médiumnité (voyance) et la guérison, servent à renforcer la foi. Finalement, nous nous pencherons sur la question de l'appartenance religieuse de ces personnes (souvent, elles ne se considèrent pas comme " spiritualistes ") et sur le rôle de l'église qui fait l'objet de notre enquête (nommée l'Église spirituelle de la guérison) dans la foi religieuse de ses membres.
Tous droits réservés @ Faculté de théologie et de sciences des religions, Université de Montréal, 2006
Ce document est protégé par la loi sur le droit d'auteur. L'utilisation des services d'Érudit (y compris la reproduction) est assujettie à sa politique d'utilisation que vous pouvez consulter en ligne.

https://apropos.erudit.org/fr/usagers/politique-dutilisation/ 


\title{
Les croyances et le croire chez des spiritualistes
}

\author{
Deirdre MeInTEL \\ Département d'anthropologie \\ Université de Montréal
}

\section{Introduction $^{1}$}

Croire est, selon la phrase de Lemieux, un «geste fondateur » de la condition humaine, par lequel le sujet se constitue en reconnaissant l'altérité, que ce soit l'Autre suprême ou les autres. Cependant, de nos jours, les croyances se basent davantage sur l' "expérience » ou sur une objectivation de la subjectivité (Lemieux 1992b, 211), que sur l'autorité ou la tradition. En même temps, les institutions religieuses perdent «leur capacité sociale et culturelle d'imposition et de régulation des croyances et des pratiques » (Hervieu-Léger 1996, 19); on observe d'ailleurs une importante diminution du nombre de leurs membres. De nombreux chercheurs constatent ainsi les provenances diverses des croyances mystiques, cosmiques et religieuses des individus, et ce, quelle que soit leur affiliation religieuse. Ils observent également le désenchevêtrement (Giddens 1990) de ces croyances de leurs sources religieuses et culturelles. Il en découle un nouveau lexique religieux constitué de descriptifs tels que "flou », "nébuleux », «à la carte », "mystico-ésotérique », etc.

À première vue, l'expérience des spiritualistes qui fait l'objet de notre enquête semble illustrer le flou informe des diverses croyances, d'autant plus que dans la congrégation que nous avons étudiée, ces croyances varient beaucoup. Par ailleurs, dans ce groupe, aucun des membres n'est né spiritualiste puisque la grande majorité est de formation catholique et entretient certaines croyances catholiques. La pratique religieuse de certains d'entre eux est également influencée par d'autres traditions spirituelles. Cependant, nous proposerons que ce flou et cette mobilité apparents n'empêchent pas

1. Nous remercions les éditeurs de ce numéro pour leurs suggestions et commentaires, ainsi que Géraldine Moissière, Isabel Heck et Typhen Ferry. 
la croyance religieuse, c'est à dire la foi, de s'épanouir dans la vie des individus que nous avons interrogés. Dans la prochaine section, nous décrivons le groupe religieux que nous étudions et nos méthodes de recherche; suivra une topologie heuristique des croyances de spiritualistes qui sont membres de l’Église spirituelle de la guérison (pseudonyme) où est centrée notre enquête. Partageant des prémisses de base avec beaucoup d'autres religions (monothéisme, croyance dans la vie éternelle de l'âme et la responsabilité de chacun pour ses actions, etc.), le spiritualisme s'en distingue par certaines croyances - on peut contacter les esprits des défunts et ces derniers peuvent venir en aide aux vivants - ainsi que par sa conception des dons spirituels dont chaque être humain serait doté. La guérison par les mains ou à distance, la clairvoyance, la clairaudience et la psychométrie (voyance à travers les objets) figurent parmi les dons possibles.

Nous essaierons de montrer que le caractère apparemment flou des croyances (la diversité de leurs provenances, la variabilité des croyances au sein du groupe spiritualiste) n'empêche pas que la croyance religieuse, soit la foi, s'épanouisse dans la vie de ces gens. Nous examinerons à cet effet de quelle manière les pratiques spécifiques au spiritualisme (par comparaison aux religions classiques en Occident), soit la médiumnité (voyance) et la guérison, servent à renforcer la foi. Finalement, nous nous pencherons sur la question de l'appartenance religieuse de ces personnes (souvent, elles ne se considèrent pas comme "spiritualistes ») et sur le rôle de l'Église spirituelle de la guérison dans la foi religieuse de ses membres.

\section{Le spiritualisme à Montréal}

Les origines du spiritualisme moderne remontent à 1847. Alors que deux jeunes filles américaines habitaient une ferme à Hydesville dans l'État de New York, elles entendirent un jour des bruits étranges. Elles parvinrent à établir un système de communication avec la source de ces bruits: un colporteur avait en effet été assassiné dans la même maison, son squelette fut retrouvé dans la cave par la suite (Aubrée et Laplantine 1990). Le mouvement qui s'est développé autour de ces deux adolescentes évolua vers la religion spiritualiste aux États-Unis et en Angleterre; en France, il influença le kardécisme ou spiritisme (ibid.). Aux États-Unis, la religion grandit dans un climat marqué par le transcendantalisme et par des personnalités telles que l'anticalviniste et philosophe métaphysique charismatique Andrew Jackson Davis (1826-1910) (Goodman 1988). Pendant plusieurs décennies, le spiritualisme fut très influent aux États-Unis, bien que le nombre de ses 
adeptes soit difficile à calculer ${ }^{2}$. Il était associé aux milieux progressistes et, en particulier, au féminisme (Braude 1989).

\section{L’Église spirituelle de la guérison et ses membres}

L'Église spirituelle de la guérison ${ }^{3}$ (ci-après ÉSG), notre terrain principal, a été fondée en 1967 par un couple de ministres spiritualistes venus d'Angleterre. En 1975, ils quittèrent le Canada et nommèrent pour remplaçant un jeune francophone, Michel. Après ce changement, l'ÉSG est vite devenue francophone, autant par sa clientèle que par la majorité de ses activités. À notre connaissance, cinq autres groupes spiritualistes existent à Montréal et se présentent comme des «Églises spiritualistes ${ }^{4}$ ». L'une d'entre elles fut fondée à peu près à la même époque que l'ÉSG, tandis que les autres auraient vu le jour plus tard. Sur ces cinq groupes, trois ont fait l'objet d'une étude de notre part: ces trois églises partagent les sept principes de base du spiritualisme, bien que leur vocabulaire varie. Parmi ces principes, on compte l'existence de Dieu (parfois appelé l' «Intelligence universelle»), la responsabilité des individus pour leurs actions, les conséquences dans l'au-delà des (bonnes ou mauvaises) actions des individus, le progrès éternel accessible à toute âme humaine, la continuité de l'existence de l'âme humaine, la communion des esprits, etc. ${ }^{5}$

L'établissement de l'ÉSG coïncide avec la sécularisation de la société québécoise, à savoir la perte de contrôle de l'Église catholique sur les institutions survenue à partir de 1960. L'ÉSG s'est donc développée dans une période de grandes mutations religieuses pour la société québécoise. Bien

2. Selon Braude, l'aversion des spiritualistes pour la formalisation institutionnelle rend difficile le calcul de leur nombre. Ainsi, les estimations de l'époque vont de quelques centaines de milliers à 11 millions $(1989,25)$.

3. Pseudonyme. En revanche, le diminutif «Église spirituelle» est souvent utilisé par les membres de la congrégation. Des pseudonymes sont également utilisés pour les individus mentionnés. Les citations d'entrevues sont basées sur le verbatim; nous avons amené des corrections mineures pour fins de clarté. Nos interlocuteurs sont souvent bilingues. Sachant que notre première langue est l'anglais, ils nous parlent en anglais parfois, d'où les citations en anglais.

4. À la différence d'autres Églises spiritualistes à Montréal, l'ÉSG jouit d'un statut légal d'église, ce qui permet à ses ministres d'unir des couples et d'organiser des funérailles.

5. Nous n'entendons pas parler de l'enfer dans le sens classique du terme, mais plutôt du fait que chaque âme sera obligée de continuer son développement dans l'au-delà. 
qu'une bonne proportion de la population qui se dit catholique reste stable entre 1960 et 1981, on observe une chute de la pratique religieuse chez les catholiques québécois, ainsi qu'un déclin vertigineux du nombre de religieux (Linteau et al. 1989, 648-649; voir aussi Bibby 1990, 135). De même, les courants protestants autrefois dominants (les Églises anglicane, unie et presbytérienne) cèdent la place à de nouveaux groupes, tels que les groupes pentecôtistes qui, eux, continuent encore à se multiplier à l'heure actuelle ${ }^{6}$ (Polo 2001).

Tributaire de moyens financiers très restreints tout comme les groupes spiritualistes américains (Zaretsky 1974, 177), cette assemblée de 175 fidèles occupe un espace loué sur deux étages. Aucun des cinq ministres (dont deux femmes) n'est rémunéré, pas plus que les guérisseurs ou les médiums qui exercent dans les services de l'église spirituelle. Les dépenses telles que le chauffage sont payées par la collecte effectuée lors des services et par les dons des membres.

Comme trois autres groupes spiritualistes, l'ÉSG se situe au centre-ville de Montréal, ce qui facilite l'accès par transport en commun auquel recourent la majorité des adeptes. Presque tous nos informateurs-clés ont grandi et habitent encore dans des quartiers ouvriers francophones de Montréal (Hochelaga-Maisonneuve, Ville-Émard, etc.). Certains d'entre eux habitent cependant la Rive Sud (Brossard principalement), tandis que d'autres qui fréquentent les offices de l'ÉSG viennent de villages assez lointains. Malgré sa situation centrale, l'ÉSG affiche une présence discrète dans la ville: elle n'a pas de téléphone et son entrée est uniquement marquée par une pancarte dans l'encadrement de la porte.

L'ÉSG n'est associée à aucune fédération, même si elle était auparavant intégrée à l'International Spiritualist Alliance dont le siège se trouve en Angleterre. Toutefois, des rapports de collaboration relient les ministres et les médiums associés aux divers groupes spiritualistes de Montréal. Les ministres ainsi que les médiums reconnus participent en tant qu'invités dans d'autres églises spiritualistes; en outre, les membres de la congrégation assistent parfois aux cultes des autres églises spiritualistes et des églises catholiques.

6. Les estimations du nombre de nouveaux groupes religieux au Québec varient entre 300 et 1000 (Bouchard 2001, 221). 


\section{L'enquête}

Pour l'auteure, spécialiste des questions de migration, d'ethnicité et d'identité, cette étude ${ }^{7}$ constitue une première incursion dans le champ de la religion, bien que nous ayons travaillé sur les fêtes religieuses capverdiennes (Meintel 2000). C’est par une série de hasards que nous avons rencontré Michel, dont les talents de guérisseur et de voyance nous avaient été rapportés. Michel, âgé dans la cinquantaine, est issu d'un milieu ouvrier francophone et il a été éduqué dans des écoles jésuites de langue anglaise. Après ses études universitaires, il a travaillé dans le milieu de la finance pour une grande entreprise; il continue d'ailleurs à travailler dans le même domaine pour son compte, parallèlement à ses activités de ministre de l'église spirituelle. Veuf puis divorcé, Michel vit actuellement une relation stable avec une médium de l'ÉSG.

Notre de prise connaissance initiale de l'ÉSG en décembre 1999 (ce, à l'invitation de Michel) nous a convaincue de l'intérêt anthropologique de ce milieu religieux ${ }^{8}$. L'ÉSG est fréquentée principalement par des Québécois de langue française, d'origine ouvrière et de formation catholique. Des pratiques telles que la voyance, la transe profonde (le channelling) et la guérison, phénomènes que nous avions associés jusqu'alors aux religions exotiques, y sont courantes. Après avoir expliqué nos intérêts anthropologiques à Michel, le médium nous a invitée à participer à un groupe "fermé "; il s'agit d'un groupe stable dont les membres sont encadrés dans le but de développer leurs dons spirituels en matière de voyance. Nous avons obtenu l'autorisation d'y mener nos recherches, à condition de conserver une certaine discrétion (à savoir, ne pas gêner l'atmosphère religieuse du groupe) et d'y participer comme n'importe quel membre du groupe. Nous avons accepté ces conditions de bon gré, puisque notre objet d'intérêt principal était l'expérience religieuse des spiritualistes. Par conséquent, nous étions disposée à conduire une enquête peu directive, basée principalement sur l'observation participante de diverses activités organisées par l’ÉSG, ainsi que sur la prise de notes ultérieure.

7. L'enquête a bénéficié d'une petite subvention CRSH, administrée par l'Université de Montréal.

8. Nous remercions particulièrement François Laplantine qui, lors de nos séjours à l'Université Lyon 2, nous a fait comprendre l'intérêt ethnologique de phénomènes tels que la voyance et la médiumnité en milieu urbain et moderne (voir, par exemple, Laplantine 1985). 
Inspirée par l'approche méthodologique de Favret-Saada (1977), nous avons adopté une démarche d'observation participante pendant presque deux ans, excepté pour la réalisation de trois entretiens semi-structurés avec Michel en 1999. Nous avons accepté d'entrer dans l'univers des significations et des relations spiritualistes, de participer aux services réguliers et au groupe fermé au même titre que les autres membres, tout en rédigeant des notes d'observations après chaque événement'. Après un an d'observations à temps partiel, nous avons commencé à entretenir des contacts informels avec quelques membres de l'ÉSG. Au cours des premiers mois d'observation, il est apparu que l'ÉSG englobe en fait plusieurs réseaux différents, chacun centré sur un ministre, ce qui a été amplement confirmé par les entrevues et par les observations subséquentes. Ainsi, très tôt dans la recherche, nous avons ajusté notre approche afin de concentrer l'étude sur Michel et sur son entourage, au lieu de faire une monographie sur l'ÉSG.

Finalement, nous avons cerné un groupe de quinze informateurs-clés qui participent ou qui ont participé au groupe fermé dirigé par Michel: il s'agit de huit femmes et sept hommes âgés de 25 à 62 ans (principalement entre 35 et 50 ans). La plupart sont divorcés et vivent maintenant des relations de couple stable; la majorité est issue de la classe ouvrière québécoise. Ils occupent des emplois réguliers; certains exercent des métiers spécialisés (par exemple, mécaniciens), d'autres travaillent dans le secteur des services, en soins à domicile, comme agent de voyage ou en tant que commis aux ventes dans une pharmacie. La plupart ont terminé l'école secondaire et quelques-uns disposent d'une formation postsecondaire en lien avec leur métier. Outre le critère d'égalité approximative entre hommes et femmes, nous cherchions des personnes impliquées dans l'ÉSG depuis au moins trois ans qui accepteraient de nous rencontrer à plusieurs reprises. Par ailleurs, nous avons été en contact et discuté informellement avec plusieurs dizaines d'autres personnes qui fréquentent l'ÉSG. Les premiers entretiens traitaient des trajectoires religieuses de nos interlocuteurs; les entretiens subséquents (toujours en cours) sont centrés sur leurs expériences des «dons spirituels », tels que la guérison et la médiumnité.

Les croyances dont il est question dans cet article n'ont pas fait l'objet de questions directes au cours des entrevues, mais elles ressortent souvent des récits des répondants. Elles apparaissent également pendant la période

9. Notre expérience personnelle de cette recherche est détaillée dans Meintel sous presse $-\mathrm{a}$. 
d'échange qui fait partie des rencontres du groupe fermé. De plus, les croyances s'expriment dans les rencontres informelles; par exemple, par la manière dont les gens racontent leurs problèmes (voir Lemieux 1992b). À l'instar de Claudia Fonseca (1991), nous avons jugé pertinent de situer les rituels et les croyances des spiritualistes par rapport à leur quotidien. En conséquence, nous avons souvent rencontré des membres de l'ÉSG en dehors du contexte religieux: dans des espaces privés comme le domicile (le leur, sinon le nôtre) ou dans les endroits publics qu'ils fréquentent (cafés, parcs et restaurants). Une telle méthodologie nous a permis de saisir la façon dont certaines croyances s'inscrivent dans la vie de tous les jours de nos interlocuteurs.

\section{Les activités de l'ÉSG}

Les activités dites «ouvertes» (au public) de l'ÉSG incluent quatre offices dont trois se déroulent le dimanche et un le jeudi soir. Les offices durent entre une heure et demie et deux heures. Tous les offices commencent par des prières d'ouverture qui incluent toujours le Notre Père (version protestante). Le service principal du dimanche se compose normalement de plusieurs hymnes (généralement des chants protestants américains ou britanniques traduits en français), d'une méditation "guidée » (un médium ou un médiumapprenti propose des images accompagnées d'une musique instrumentale Nouvel Âge), de l'offrande (la collecte d'argent), de la bénédiction de l'offrande et d'une période de «messages » (clairvoyance donnée par un ou plusieurs médiums à des individus dans l'assistance); il se termine par la prière et l'hymne de fermeture. Les dimanches où Michel fait l'office, la méditation est remplacée par un discours, car il aime prêcher; ses «dissertations» (le terme classique spiritualiste) sont d'ailleurs très appréciées de son auditoire. L'office principal du dimanche est suivi par un "office de guérison » au cours duquel un ou plusieurs ministres et leurs assistants donnent la guérison par l'imposition des mains. Normalement, les guérisseurs ne touchent pas le corps; ils travaillent le «champ énergétique» autour de l'individu qui est assis, les yeux fermés. Leur travail ressemble quelque peu à celui des «magnétiseurs » décrits par Acedo (2000). L'office de guérison est, lui aussi, marqué par des prières d'ouverture et de fermeture et par la collecte et par la bénédiction de l'offrande; il est également accompagné d'une musique de style Nouvel Âge. Vers la fin de l'après-midi, un «office de messages » plus court, sans sermon, parfois sans méditation et incluant moins d'hymnes, a généralement lieu. Le service du jeudi est similaire au troisième office du dimanche. À la différence des groupes observés par Zaretsky 
(1974), l'ÉSG possède un inventaire d'artéfacts modeste, mais significatif : un petit orgue, des tableaux qui représentent des thèmes spirituels de style Nouvel Âge, une grande Bible (version King James), etc. Pour les offices des grandes fêtes, les ministres mettent des vêtements rituels, une étole par exemple.

Lors des offices, le ou les médiums ${ }^{10}$ s'adressent aux individus de l'assistance en leur donnant des messages de clairvoyance qui peuvent aussi bien porter sur la santé que sur la vie émotionnelle, amoureuse ou familiale. Normalement, les médiums ne connaissent pas ou peu la vie privée des gens qu'ils interpellent. Les messages touchant les relations personnelles sont transmis avec discrétion, comme: «Ça brasse au travail pour toi! Pas facile! $\mathrm{Tu}^{11}$ vas avoir à vider ton sac bientôt avec un collègue, un homme...» En matière de santé, les médiums prennent soin de ne rien prescrire, mais simplement de suggérer ${ }^{12}$, par exemple: "Je vois beaucoup d'oranges autour de vous, donc essayez d'en manger plus; c'est une suggestion seulement. » Parfois, le médium observe un ou plusieurs esprits autour de l'individu auquel il s'adresse; dans certains cas, il s'agit de religieux catholiques «Je vois une religieuse, une membre de la famille» — ou de la Vierge Marie.

De temps à autre, un "office de transe » est offert au cours duquel l'un des médiums entre dans une transe profonde, ce qui permet à un ou plusieurs "esprits qui veillent sur les gens et essaient de les guider» de parler à travers le médium. Précisons que la glossolalie (Goodman 1972; Samarin 1972) ne se produit ni chez les médiums ni dans l'assistance, à la différence de groupes pentecôtistes (Goodman 1972) ou charismatiques (Csordas 2001), par exemple. Par ailleurs, les « dons » connus des spiritualistes (guérison, voyance de différentes sortes) ne sont pas le signe du mérite spirituel comme peut l'être la glossolalie pour les pentecôtistes (Goodman 1972). Au contraire, dans l'optique spiritualiste, tout le monde possède des «dons » (de guérison ou de voyance) qui peuvent être développés par la pratique. Mais, il est important que ces dons soient encadrés spirituellement. À ce propos, Michel appelle souvent les gens à se méfier des «ésotériques » qui utilisent leurs dons de voyance à des fins égoïstes (gain matériel, pouvoir sur les autres, etc.). D'autre part, il insiste sur la «discipline dans la voyance » : il s'agit de ne pas utiliser la voyance pour animer une soirée entre amis, par

10. Parfois plusieurs médiums travaillent ensemble.

11. Le tutoiement est habituel entre les gens de l'ÉSG qui se connaissent.

12. Comme Michel et d'autres médiums le rappellent souvent à leurs élèves, la loi interdit les prescriptions en matière de santé par des non-médecins. 
exemple. Par ailleurs, il explique que certains médiums, même parmi ceux ayant été encadrés par lui ou d'autres ministres, développent parfois de "gros ego » : ils deviennent vaniteux et oublient la source divine de leur don. Ils sont d'ailleurs éventuellement obligés de cesser de pratiquer la voyance pour cause de problèmes personnels. La voyance, selon Michel et d'autres ministres de l'ÉSG, doit servir principalement à aider les gens dans leur vie spirituelle ainsi que dans celle de tous les jours.

Du fait de la composition de l'assemblée, les offices ont généralement lieu en français, bien qu'il y ait un ou deux offices bilingues par mois depuis 2003. Un service mensuel en anglais a été inauguré récemment. La plupart des médiums et apprentis sont bilingues et transmettent les messages en anglais à l'occasion. L'office principal attire souvent une cinquantaine de personnes, voire plus; à l'inverse, parfois seulement une douzaine de membres sont présents, selon le ministre, la période de l'année ou la météo. D'après nos calculs, la proportion d'hommes correspond habituellement à environ un quart de l'assemblée. On remarque une prépondérance de gens d'âge moyen, entre 30 et 55 ans.

Les groupes (ou cercles, dans le vocabulaire spiritualiste classique) fermés, appelés "cours de développement spirituel », se réunissent dans les locaux de l'ÉSG (comme celui que nous avons fréquenté), chez le médium ou chez un membre du groupe. Les membres de ces groupes stables s'engagent à participer à toutes les rencontres qui sont bimensuelles ou (pour un des groupes) hebdomadaires. Le médium choisit des participants (il y a parfois plus de demandes que de places) pour former un groupe dont le nombre ne dépasse pas 17 ou 18 personnes. Les membres paient chaque semaine des frais modestes ( 8 \$ actuellement) qui, selon Michel, permettent même aux personnes sans emploi de participer. Dans ces groupes, les élèves sont initiés à divers types de voyance, laquelle n'est qu'un outil dont le développement doit approfondir la spiritualité de l'élève, nous dit Michel.

La rencontre débute avec une prière d'ouverture où les participants se tiennent la main, en formant un cercle. Puis ils se livrent à des exercices de respiration tout en visualisant un soleil au-dessus de la tête "pour ouvrir les chakras " et demander la protection spirituelle. Vient ensuite la méditation guidée par Michel, elle dure environ vingt minutes et se déroule dans une pièce éclairée par une lumière bleue. Suite à cette période de méditation, chaque participant parle de ce qu'il a «reçu».

Après une pause, la rencontre se poursuit par une période dite de «méditation active », à savoir l'initiation à la voyance. À ce moment, l'éclairage 
devient rouge, dans le but de stimuler les capacités de voyance des participants. Généralement, Michel précise l'exercice, par exemple: "Vois une couleur pour les trois personnes à ta gauche et explique ce qu'elle signifie.» Chacun raconte ensuite ce qu'il a capté et pour qui. En général, Michel s'abstient de commenter les résultats, se limitant à des encouragements comme: «Très bien, merci beaucoup!" Enfin, une prière au cours de laquelle les personnes forment un cercle en se tenant à nouveau les mains vient clore la session.

D'autres groupes spiritualistes montréalais ou britanniques soulignent davantage la validité objective des contacts établis avec le monde des esprits, comme Aubrée et Laplantine (1990) l'observent chez les kardécistes français. Pour notre part, nous n'avons jamais entendu de références à des esprits illustres (comme chez les kardécistes, par exemple); de plus, les médiums ne tentent pas d'identifier les esprits qu'ils aperçoivent, ils essaient plutôt de transmettre leur message à l'intéressé. Malgré les différences d'approche entre les différents ministres de l'ÉSG, nous constatons qu'ils partagent un certain nombre de prémisses. Outre les sept principes spiritualistes mentionnés auparavant, ils présentent le spiritualisme avant tout comme une religion, plutôt que comme une "science » ou même une "philosophie de la vie », contrairement aux autres groupes spiritualistes de Montréal.

Notons que le mot religion est rarement utilisé par les membres de l'ÉSG, malgré le fait que, dans ses discours devant la congrégation, Michel insiste sur le statut du spiritualisme en tant que religion. Sans contredire Michel, les membres que nous avons rencontrés préfèrent employer des termes tels que spiritualité ou cheminement spirituel. Pour sa part, Michel accepte bien (et le dit dans ses discours publics) que certains préfèrent appréhender le spiritualisme comme approche philosophique ou psychologique.

Bien que le spiritualisme provienne de la tradition protestante, ni les membres ni les ministres ne se considèrent comme "protestants ", et encore moins comme "chrétiens". Alors que nous nous interrogions sur la présence d'une Bible dans l'église, Michel nous expliquait que pour obtenir le statut légal comme église, il était obligatoire de reconnaître un livre sacré. Étant donnée la composition majoritairement catholique du comité décideur, la Bible constituait un choix pratique. Lors des cultes, Michel commence souvent ses discours par une phrase de la Bible. À d'autres moments, il se réfère à la Bible comme à une partie de notre tradition culturelle et s'empresse de souligner l' " ouverture » du spiritualisme à d'autres textes sacrés, tels que le Bhagavad-Gita et le Coran. 


\section{Affiliations et appartenances}

Comme nous en avons discuté ailleurs (2003), devenir membre n'équivaut pas à se convertir. Il s'agit plutôt de contribuer financièrement aux frais de l'église, à raison de $20 \$$ par an, ce qui permet à l'individu de participer à certaines activités, emprunter des livres de sa bibliothèque, etc. D'autre part, chez les spiritualistes, il n'y a pas de conversion. Par ailleurs, nous n'avons connu aucun cas de baptême d'adultes, puisque, selon Michel, «ils sont déjà baptisés ». Le prosélytisme n'est pas encouragé, ni même l'encadrement spiritualiste des enfants; on ne baptise pas les enfants et aucune activité n'est prévue pour ces derniers. Le recrutement passe davantage par le réseau des adeptes; ainsi, on peut trouver plusieurs membres d'une même famille, mais jamais de familles entières. Parfois, certains adultes invitent un de leurs parents; le plus souvent, l'entrée dans l'église passe par un ami, un collègue de travail ou un voisin.

Les membres participent aux activités de l'ÉSG de façon très variable. Certains fréquentent des groupes fermés, mais ne se rendent jamais aux cultes, les trouvant «trop religieux »; d'autres, plus âgés, vont aux services de l'église, mais ne s'intéressent pas aux activités des groupes fermés. D'autre part, il arrive que des gens entretiennent une autre affiliation religieuse tout en participant aux activités de l'ÉSG (Meintel 2003). Plusieurs membres qui sont très actifs dans l'église comme guérisseurs ou comme médiums s'associent également à des activités liées au spiritualisme autochtone, par exemple, les tentes de sudation (sweat lodge), le néochamanisme, la Danse du Soleil aux États-Unis, etc. La majorité des membres de l’ÉSG est née et a été élevée dans la religion catholique, y compris Michel. C'est ainsi que le vocabulaire catholique (par exemple, l'utilisation du mot messe au lieu de service) constitue une base commune. Bien que nous n'ayons rencontré personne qui maintienne une pratique orthodoxe du catholicisme (la messe dominicale et la confession), nous n'avons pas entendu parler de «renoncement » à cette religion. Au contraire, beaucoup continuent à entretenir des contacts occasionnels avec les milieux catholiques, surtout pour les grandes fêtes religieuses, en particulier Noël. Implicitement, l’ÉSG reconnaît d'ailleurs la place toujours dominante du catholicisme dans le milieu environnant. Ainsi, les offices de Noël et du Nouvel An n'ont jamais lieu le même jour que ces fêtes, mais quelques jours auparavant. Comme nous le montrerons plus loin, l'expérience du catholicisme représente un point de référence partagé et une gamme de ressources symboliques qui sont parfois activés dans le contexte spiritualiste. 


\section{Les croyances des spiritualistes}

Selon Lemieux, nous possédons tous des croyances et le croire constitue un "des gestes fondateurs de l'être humain» (1992a, 50). L'auteur montre également que les croyances religieuses peuvent se mêler à des croyances d'autres provenances, à l'instar de la réincarnation qui serait une idée acceptable pour un quart ou plus de la population québécoise (Lemieux 1992a, 26). Ici, nous esquisserons une «topologie » heuristique des croyances des spiritualistes que nous avons rencontrées. Sans statuer sur le caractère religieux de toutes ces croyances, précisons qu'il s'agit grosso modo, du religieux proprement dit (l'Alterité transcendante), du cosmique, de l'âme et de la médiumnité. Il s'agit de croyances religieuses dans la mesure où elles concernent Dieu, les dons spirituels, la relation avec Dieu et ce que mes interlocuteurs appellent la «vie spirituelle».

Dans la première catégorie, on trouve les croyances qui sont le fait de tous les spiritualistes que nous avons rencontrés. La deuxième concerne des croyances qui sont moins généralisées et moins centrales dans le spiritualisme; souvent, les croyances de cette catégorie proviennent d'autres traditions, mais elles sont présentes dans le quotidien de l'ÉSG; elles sont parfois mentionnées dans les messages (clairvoyance) donnés par les médiums et dans les échanges du groupe fermé. Une troisième catégorie englobe la pléthore de croyances, souvent inconnues pour beaucoup de membres, auxquelles certains souscrivent et d'autres non.

\subsection{Croyances généralisées}

Les sept «principes » du spiritualisme sont considérés comme des prémisses, mais il est souvent dit par les ministres qu' "il ne s'agit ni de credo ni de dogmes » et que l'église est ouverte à toutes les traditions spirituelles. Michel et les autres ministres évoquent ces principes, tout en précisant qu'ils peuvent être interprétés de diverses manières. Ceux-ci incluent l'existence de Dieu (parfois appelé l' «Intelligence universelle»), la responsabilité de l'individu pour ses actions, les conséquences de ses (bonnes ou mauvaises) actions qu'il doit subir dans l'au-delà, le progrès éternel ouvert à toute âme humaine, la continuité de l'existence de l'âme humaine, la communion des esprits, etc. Dans le spiritualisme tel que pratiqué à l'ÉSG, la « communion des esprits » est interprétée plus littéralement que dans le catholicisme: elle désigne la possibilité offerte à plusieurs, de rentrer en contact direct avec les esprits des morts (ceux qui ont passé à « une autre dimension») et surtout avec ceux 
qui sont perçus comme «évolués » et qui fonctionnent comme des guides pour les vivants (sur terre).

Ces sept principes sont généralement acceptés par les églises qui se disent spiritualistes, ainsi que par l'International Spiritualist Federation ${ }^{13}$, dont le siège se trouve en Angleterre. Toutefois, le vocabulaire peut varier et certains groupes spiritualistes évitent d'utiliser le discours religieux en se référant davantage à une «science » ou à une «philosophie de la vie ». À l'ÉSG, à l'inverse, le spiritualisme est présenté comme une religion et le langage fait souvent référence à une sous-strate religieuse catholique partagée par la majorité des membres ${ }^{14}$. Quant il dirige l'office, Michel demande souvent à la congrégation de lire ces principes à haute voix, justement pour souligner le statut d'église de l'ÉSG. Il insiste également sur son statut légal qui permet aux ministres de prononcer des mariages (y compris entre homosexuels) et de diriger des funérailles.

Outre les sept principes, il existe des croyances qui semblent universelles chez les spiritualistes que j'ai rencontrés, notamment la croyance en l'existence des anges et des esprits évolués qui nous guident dans la vie terrestre ${ }^{15}$. En outre, ils croient que nous possédons tous des dons spirituels (guérison et voyance de différentes sortes, projection astrale, etc.) qui peuvent être développés et utilisés pour faire le bien ou le mal. Enfin, ils pensent que la guérison spirituelle des maux physiques et psychiques (ces derniers étant prédominants chez ceux qui recherchent la guérison) est possible. Par ailleurs, ces spiritualistes croient aussi à la présence d'esprits «portiers » (gatekeepers) qui constituent les guides principaux de l'individu. (Le portier est celui qui permet aux autres esprits de parler à travers le médium dans la transe profonde, ou channelling.) Très souvent, le guide principal est un esprit d'autochtone, thème que nous espérons développer ailleurs. Finalement,

13. L'adresse Internet de cette fédération est $<\mathrm{http}$ ://www.isfederation.org $>$.

14. Sur la première page de l'hymnal de l'ÉSG, avant les sept principes on trouve :

Le spiritualisme est une religion fondée sur la philosophie de la bonté humaine, la connaissance spirituelle et des faits scientifiques [...] il nous donne la force et la patience de rendre notre vie plus productive et en accord avec les idéaux du Divin Père. Le spiritualisme n'a ni dogme ni credo proprement dit, mais il s'appuie sur sept principes...

15. Un des principes tel qu'écrit dans l'hymnal de l'ÉSG se lit: «... la communion des Esprits et le ministère des Anges ". 
nos interviewés croient que les animaux ont des âmes et qu'ils continuent à vivre dans l'au-delà ${ }^{16}$.

Du côté négatif, les spiritualistes que nous avons rencontrés croient aux «mauvaises entités ", soit aux esprits de morts peu évolués qui font mal aux gens, qui les attaquent en les détournant de leur voie spirituelle ou, plus rarement, qui essaient de les entraîner dans une dépendance sexuelle. Nous avons entendu des discussions au sujet de tels phénomènes dans le groupe fermé, parfois à l'instigation de Michel.

Soulignons que les croyances de cette catégorie relèvent de l'expérience vécue de mes informateurs, sinon de leurs proches. Beaucoup ont vu des esprits et des anges, d'autres ont vu ou senti la présence de leur animal domestique décédé, et plusieurs disent qu'ils ont été attaqués par des mauvaises entités. En réalité, le fait de croire aux guides et à la guérison spirituelle est essentiel au déroulement des groupes fermés, ainsi qu'à la médiumnité telle qu'elle se présente dans l'ÉSG. Les rêves prémonitoires font partie, eux aussi, des croyances et expériences communes à mes interlocuteurs. (Parfois, dans la voyance les médiums signalent aux individus qu'ils ont fait ou vont faire de tels rêves). Les croyances en l'astrologie, les cristaux et le tarot sont également généralisées, ainsi que le reiki. Les gens rencontrés disent les avoir expérimentés et y croire, mais ils ne les confondent pas avec leur vie spirituelle. Comme le dit Sylvie, une réceptionniste dans la soixantaine qui travaille comme médium et guérisseuse à l’ÉSG:

Il faut toujours dire qu'il y a une différence entre l'ésotérisme et la spiritualité. Et la boule de cristal, les cartes et tout ça, c'est de l'ésotérisme. Donc, les gens font ça souvent pour le pouvoir de la chose, ou pour la capacité qu'ils ont de prouver à quelqu'un qu'ils ont ce don-là, (inaudible) et aussi pour faire de l'argent ou [...]. Il y a quand même une différence de le faire de cette façon-là que de le faire spirituellement, de s'en servir spirituellement.

\subsection{Croyances répandues}

La seconde gamme de croyances rassemble les idées qui sont largement acceptées par les spiritualistes que nous avons rencontrés, mais pas nécessairement par tous; elles sont un peu moins centrales aux pratiques de l'église que la première catégorie et semblent relever surtout d'autres influences religieuses. Beaucoup croient à la réincarnation, tout comme les Québécois

16. Cette croyance paraît assez ancienne et généralisée en spiritualisme (voir, p. ex., Barbanell 1940). 
catholiques étudiés par Lemieux (1992b), mais Michel et d'autres membres n'en sont pas convaincus. D'autres notions venues de l'Orient par le biais des approches Nouvel Âge, telles que l'idée de chakras, sont souvent mentionnées et Michel les intègre fréquemment à son discours. Plusieurs nous ont parlé de gens qui font de la «projection astrale » (se projeter ailleurs, en dehors de son corps physique), mais disent ne pas l'avoir expérimenté euxmêmes. De la même façon, concernant ce que nous appelons la sous-strate de croyances catholiques, non seulement Michel, mais aussi d'autres médiums évoquent la Vierge Marie ou le frère André (deux figures vénérées par bien des membres plus âgés) au cours des messages adressés à des membres de la congrégation. Par exemple: "Je vous vois devant une dame habillée en bleu, vous êtes dévouée à la Vierge Marie... » Plusieurs membres du groupe fermé gardent précieusement un chapelet qui leur a été donné par une grandmère, une tante...

Lors d'une conférence donnée à l'église sur la vie après la mort, Michel a évoqué l'existence, dans "l'au-delà ", de plusieurs niveaux, au sein desquels les esprits s'adonneraient à diverses occupations qui se comparent aux activités terrestres. La femme qui est alors assise à côté de nous est membre de l'église depuis plusieurs années; elle nous glisse: « Je ne suis pas sûre que je crois à tout ça. » Même Michel affirme qu'il n'est pas convaincu par l'idée de la réincarnation, bien que la majorité des spiritualistes qu'il connaît y croient. «J'espère, dit-il, que ce n'est pas vrai, car j'ai vu d'autres royaumes tellement plus merveilleux. » Ces désaccords au sujet des croyances moins centrales au spiritualisme sont acceptés sans créer de divisions plus profondes.

\subsection{Croyances idiosyncratiques}

La troisième catégorie inclut des croyances peu connues de la majorité des membres, mais auxquelles souscrit l'un ou l'autre individu. Par exemple, si on laisse un verre d'eau à côté de son lit pendant la nuit, au petit matin, les esprits l'auront infusée de leurs bonnes énergies — le mot énergie est d'ailleurs très courant dans le discours de ces spiritualistes, tout comme le mot vibrations. Également, si la flamme d'une chandelle s'éteint subitement, ce peut être l'œuvre d'un mauvais esprit. Certains croient fermement aux extraterrestres, mais d'autres n'en parlent pas. Cette catégorie regroupe donc des croyances qui ne sont pas considérées comme centrales à la vie spirituelle. 


\section{Les croyances et la croyance}

Nous constatons que les croyances des spiritualistes touchés par notre enquête sont plutôt variables et incluent certaines notions associées au Nouvel Âge. Cependant, nous sommes loin de la «nébuleuse mystiqueésotérique » observée par Champion (1990,1991); des pratiques telles que l'astrologie, le tarot et l'utilisation des cristaux sont vues diversement comme amusantes, parfois fiables, parfois bénéfiques, mais pas spirituelles en soi. Ailleurs, nous avons discuté les influences des courants "Nouvel Âge » sur les pratiques spiritualistes (par exemple, la méditation pratiquée dans le groupe fermé et la méditation guidée qui fait partie de certains services à l'église). Cependant, Michel souligne l'écart entre l'ésotérisme, qu'il considère néfaste, et le spiritualisme. Il déconseille à ses élèves la fréquentation des foires «psychiques » et considère dangereuse l'utilisation des planches ouija. Selon lui, la voyance des ésotériques est souvent utilisée pour manipuler ou pour satisfaire l'appât du gain. Par contre, la voyance des médiums spiritualistes est censée rendre service aux gens dans le cadre de leur vie "matérielle », les attirer et éventuellement les aider dans leur évolution spirituelle. «La voyance, c'est comme les vitraux d'une église. [...] Ils attirent les gens, à l'église, au spiritualisme. C'est pour les attirer, pour les aider dans leur évolution. » (Michel)

Bien que les croyances varient d'un individu à l'autre et qu'elles puissent inclure des éléments non-religieux, les propos des personnes interrogées se ressemblent à plusieurs égards. Les croyances les plus importantes dans le spiritualisme, selon leurs dires, relèvent de leur propre expérience: l'existence de Dieu et le contact avec les esprits font partie du «su» (Pouillon 1993). En fait, le terme «croyance» traduit mal le type de représentations dont il s'agit. Comme explique l'auteur, le mot «croire» connote le doute et la relativisation. Or, nos interlocuteurs considèrent qu'ils ont fait l'expérience de Dieu (par leurs prières exaucées et, parfois, par des expériences extatiques) et des esprits (que plusieurs d'entre eux ont vus et que la majorité a sentis). Cependant, comme le constate Milot $(1992,119)$ au sujet des croyances des Québécois, la validation empirique n'est pas nécessaire à l'adhésion. Bon nombre de spiritualistes n'ont jamais vu d'esprits, mais ils y croient fermement. L'expérience des "guides ", tout comme l'expérience de Dieu, peut passer par la prière, et par le sentiment que les prières sont entendues et souvent exaucées.

Nos observations à long terme nous ont permis de voir que Dieu et les esprits guides sont présents dans le quotidien de nos interviewés et dans 
leurs interactions sociales. Rappelons qu'ils sont engagés dans la voie spiritualiste depuis plusieurs années. Pour eux, les difficultés de la vie - le chômage, les dettes, le divorce, les difficultés avec les enfants, etc. - sont autant d'occasions de prières. La voyance des médiums leur fournit un soutien à l'instar de Pierrette qui, au début de la cinquantaine, a vécu une longue période de chômage et qui a souvent reçu des «messages » indiquant qu'elle trouverait un emploi durable. Elle explique: «À la rigueur, les messages, je peux m'en passer. Des fois ça le rend plus dur d'attendre. » En fait, pendant longtemps, elle a prié intensément pour être aidée et guidée. Pierrette était alors convaincue que ses guides l'amèneraient à un emploi dans le secteur où elle voulait travailler, celui des voyages, et ce, en dépit de son âge, de son manque d'expérience pertinente et du climat économique très défavorable dans ce secteur. Son projet s'est réalisé après plus de trois ans de quête.

Nancy, âgée de 35 ans, raconte qu'elle a trouvé une ambiance «négative » dans le bureau où elle a commencé à travailler comme réceptionniste; elle s'est empressée de la «nettoyer » spirituellement à l'aide de ses guides. Elle raconte: "Oui, je l'ai demandé pour moi en premier. Me first! Me first! Mais je me dis, les gens autour de moi aussi, ils arrivent avec plein de bagage eux autres aussi; comme moi, j'arrive avec du bagage [...]. Ça fait que c'est quelque chose que les autres vont y goûter. Puis maintenant c'est correct. Tout simplement correct [...]. Mais des fois ça peut prendre de l'énergie...»

En dehors des situations difficiles, l'ordinaire de ces individus est caractérisé par un dialogue constant avec Dieu et avec leurs guides. Comme Pouillon l'observe chez les Dagealat (1993), les esprits font partie du monde des vivants, invisibles pour la plupart, mais présents. Roger, âgé de 64 ans, travaille actuellement comme inspecteur pour des épiceries. Depuis huit ans, à la suite de sa rencontre avec un chamane algonquin à Québec, il poursuit un apprentissage du chamanisme autochtone. "For me both of them are together [...]. Everything comes to the same place, medicine Tibetan, Charismatics [...]. There's only one God, call Him the way you want.» Quand nous lui demandons s'il observe une pratique spirituelle en dehors des cultes, il répond:

I pray all day long, no special time [...] driving my truck, I don't even listen to music in the truck [...]. To give you an example, I was coming here this morning, so I kneel down and I ask God to tell you everything I knew from the beginning till now $[\ldots]$ and try to be as simple as that, not to put more things for show, just what's supposed to be there [...]. After this I'll sit in my 
truck and say thank you for what happened this morning [...]. Sometimes going from Montreal to X it's an hour, I repeat for the hour: « Merci Jésus de m'accompagner ", like a mantra, or «Saint Joseph, priez pour moi, exaucez ma prière ». It's always working. Touch wood, everything I ask for, I have it.

Revenons à Nancy, déjà citée plus haut, elle exerce une deuxième activité comme massothérapeute. Elle raconte:

Quand je fais du massage, c'est de la guérison, une forme de guérison que je fais. Je t'ai parlé du livre tantôt, Le Pouvoir bénéfique des mains, que j'ai comme ouvert à quelques pages, puis là je lisais : ah wow! Ah wow! Puis à un moment donné, j'arrive à une image; il y a une personne allongée sur la table et la personne qui fait la guérison et là tu vois toutes les aides en arrière, les guides. J'ai fait: this is what I feel when I... quand je masse. Je ressens les présences qui sont là, parce que des fois je vais faire des massages et j'ai comme l'impression qu'on prend mes mains et qu'on me guide.

En fait, ce qui distingue ces spiritualistes d'autres Québécois, ce n'est pas forcément la nature de leurs croyances, dont les plus importantes sont partagées par bon nombre de non-spiritualistes (voir Lemieux et Milot 1992) et dont les autres sont assez variables, mais plutôt la fermeté de leur croyance, autrement dit, la qualité de leur foi. Leurs croyances, vues de l'extérieur, peuvent ressembler à une «religion à la carte» (Bibby 1990), ou même à une «mystique-ésotérique »(Champion 1990) liée à leur variabilité et à la diversité de leurs provenances. Pourtant, si nous écoutons les propos de nos interlocuteurs, leur foi semble très solide.

Les membres de la congrégation de l'ÉSG que nous avons rencontrés rappellent qu'ils étaient croyants avant de connaître le spiritualisme. La plupart avaient cessé de pratiquer le catholicisme, sans toutefois le renier. Michel confirme que, en général, les gens attirés vers l'ÉSG sont déjà croyants. On dirait qu'ils étaient «believers without belonging " selon la phrase de Davie (1990). En général, c'est une crise de la vie - un divorce, un décès, une perte d'emploi subite, parfois l'alcoolisme - qui les pousse vers une quête personnelle. Cependant, la quête qui les amène à l'ÉSG, normalement à l'invitation d'un de leurs proches, prend forme après une crise qui a secoué ou brisé les repères qui ont structuré leur vie, les laissant avec un besoin vif de (re)donner un sens à leur vie. Le retour au catholicisme leur paraît inconcevable. En effet, comme bien d'autres Québécois, l'autorité religieuse n'est une source ni de valeurs ni de légitimité (Lemieux 1992b, 207), et leur image de l'église catholique demeure celle d'une religion autoritaire et dogmatique. Pour la même raison, les églises évangéliques ne les attirent pas davantage; certains 
membres qui ont été en contact avec ces mouvements considèrent qu'elles prétendent détenir la vérité. Blaise, née en Afrique de l'Ouest, est au début de la cinquantaine et il a visité une église évangélique qui rassemblait beaucoup d'Africains: "Je peux te dire que je n'ai pas été bien à l'aise. Parce que... c'est comme dire que c'est le pentecôtisme qui est le bon. C'est comme oublie ce que tu es, et c'est ça la Vérité [...] j'ai l'impression qu'ils veulent m'entraîner à cette conversion [...]. Je sens ça, même s'ils ne le disent pas, je sens ça, et moi j'attends. »

\section{Croyance et expériences extraordinaires}

Il est pertinent de s'interroger sur les pratiques qui distinguent les spiritualistes de la voyance médiumnique et de la guérison spirituelle: quel est leur rôle par rapport aux croyances, et surtout par rapport à la foi religieuse qui caractérise les spiritualistes que nous avons rencontrés? Rappelons que ces individus ont été initiés à la voyance, sinon dans le groupe fermé, au moins aux services de l'église, et qu'à l'occasion, ils ont reçu la guérison (par l'imposition des mains); plusieurs sont d'ailleurs guérisseurs dans le service de guérison du dimanche. La guérison, comme la voyance, oblige à un lâcher prise tant pour celui que la reçoit, que pour celui qui la transmet.

L'efficacité de ces pratiques encourage les uns et les autres à les poursuivre et ainsi à éliminer le doute qu'elles induisent. Ceux qui reçoivent la guérison ressentent généralement un bien-être accru (à part les quelques cas plus spectaculaires de cures de maladies physiques attribuées à la guérison). Cependant, pour la ressentir, il faut au minimum entretenir une foi « interpersonnelle ( Welte 1984, 60) en les capacités du guérisseur, c'est-à-dire une ouverture envers lui. (Soulignons que, le plus souvent, les guérisseurs ne connaissent pas les individus qui reçoivent la guérison, ni les médiums ceux à qui sont destinés leurs messages.) La voyance des médiums à l'égard de la nature des problèmes qui préoccupent leurs interlocuteurs s'avère généralement juste, ce qui est rassurant pour ces derniers. Ainsi, les médiums consolident l'appui et l'encouragement qu'ils essaient de transmettre aux individus auxquels ils s'adressent. Parallèlement, les prévisions qui composent une partie des messages, qu'elles soient optimistes ou pas, sont perçues comme des orientations d'autant plus solides.

Transmettre la guérison, transmettre des messages aux autres constituent généralement autant d'occasions d' " actes de foi ». L'initiation à la voyance reçue dans le cadre du groupe fermé incite à éliminer ses propres doutes, car typiquement, le débutant a tendance à considérer les perceptions qui lui 
parviennent comme étant «juste de l'imagination». Avec la pratique, il acquiert de la confiance et reçoit souvent la confirmation des autres membres du groupe à qui ces messages sont destinés. Quant aux guérisseurs, leur travail les amène en général à l'oubli du soi et au contact avec «l'énergie de la guérison ». Dans les deux cas, les acteurs se considèrent comme des véhicules, de simples intermédiaires; normalement, ils remercient leurs guides et leurs anges de les avoir aidés à transmettre la voyance ou la guérison, qui sont toutes deux de source divine de leur point de vue. L'expérience de la médiumnité et celle de transmettre (ou de recevoir) la guérison ne sont pas la base de la foi, mais elles la renforcent continuellement. Par ailleurs, ces expériences gagnent en profondeur et en puissance émotionnelle, puisqu'elles s'inscrivent dans le corps (voir Cataldi 1993 sur le lien entre l'embodiment et l'émotion).

Pour des raisons d'espace, nous approfondirons la question de l'incorporation ultérieurement; nous nous contenterons ici d'affirmer que selon nos observations et entrevues, la voyance pratiquée dans le groupe fermé passe souvent par des sensations corporelles (chaleur, froid, douleur, etc.); de telles sensations inspirent des flashs par lesquels on capte ce qui se passe chez l'autre. Les guérisseurs, par exemple, peuvent ressentir des picotements ou de la chaleur dans les mains. D'autre part, les voyants parlent fréquemment de douleurs ressenties lorsqu'ils centrent leur attention sur l'autre (par exemple, mal aux jambes). Il leur arrive de «voir» des anges, des auras, des guides, que ce soit de manière physique (comme des apparitions) ou "dans l'esprit» (image mentale), et d'entendre des mots et des phrases, physiquement ou mentalement (voir aussi Meintel sous presse - a et b).

Marie, quadragénaire et ancienne enseignante, sort d'un long congé de maladie. Elle était catholique pratiquante dans sa jeunesse et a connu le spiritualisme il y a plus de vingt ans. Depuis environ trois ans, elle travaille comme médium et guérisseuse à l'ÉSG.

Moi, je suis beaucoup dans le senti [...] je veux dire peu importe où t'es, la guérison, tu la transmets, la clairvoyance, bien là, à ce moment-là, les endroits où je vais recevoir pour donner la clairvoyance [...]. Je reçois pour moi quand je suis en travail. Je veux dire, je reçois pour moi-même. Dans mes travaux, puis là on me dit, "Marie, il y a ça, ça, ça ", quand mes anges et mes guides de Lumière travaillent avec moi, je reçois comme je te regarde là, c'est comme l'écran qui se place devant moi et là je reçois, j'entends, je sens, il y a des images, il y a des symboles qui s'installent [...]. Pour me guider [...] parce que c'est toujours dans un but de servir l'humanité. Bien je reçois dans un but de servir [...] (pour) m'aider à m'aider pour aider les autres. 
Blaise est un des guérisseurs de l'église. Né en Afrique de l'Ouest, il a grandi dans la foi catholique. Il mentionne la proximité entre l'animisme africain et le spiritualisme, "dans le sens où, dans le spiritualisme, tout est la création de Dieu, alors tout est animé de quelque chose. » Quand il transmet la guérison:

Je fais la prière au début, je reste en état d'écoute ou en mode méditatif, et quand je sens venir la distraction [...], je prends des respirations et je mets ma conscience en connexion. C'est ça, je ne veux pas faire le médecin qui veut diagnostiquer ou qui veut faire quoi que ce soit. Je transmets ce que la personne a besoin, donc je me ramène, soit en respiration, en pensant à mon (enracinement). Et des fois [...] et c'est à travers ça que j'ai le... j'essaie... ou je me connecte à ce que je dois faire, la personne des fois, ça peut être plus sur le cœur ou sur la rate, qu'importe, ici, bon... moi je m'abandonne [...]. Je m'abandonne d'être guidé...

Pour sa part, quand Roger transmet la guérison: «Je prie, mon Dieu, laisse ta lumière pénétrer à l'intérieur de cette personne. Guéris-le de toute inharmonie. Anything that's not good for him, take it out [...]. This is a gift that He gave me and I have to share it with the people." Quand il donne la voyance à l'église (il ne le fait jamais à l'extérieur): "I'm in like a trance, but not completely gone, you know? I know what I'm doing, but it's to open, let the spirit come in, and the guides from the other people. Their guide and my guide to send them what they need.

Marie, citée ci-haut, parle de son activité de guérisseuse:

Moi, quand je transmets, je suis tellement dans un état second à ce momentlà, que $[\ldots]$ je remets tout entre les mains de Dieu, les anges, (et) je me dis: «Ils sont entre bonnes mains. » [...] J'ai cette paix-là qu'on s'occupe d'eux [...]. Je sens que je capte les larmes, je capte les symptômes, mais je me dis si en tant que canal je peux aider à enlever [...]. La paix s'établit parce que je sais que la guérison travaille pour eux puis [...] ils sont entre les mains de Dieu à ce moment-là [...]. On devient comme en communion avec le tout. Cette communion-là de la guérison, on la reçoit et on sent que ça la transmet à l'autre aussi. Cette fusion-là, je la sens. C'est comme, je sens une grande force à l'intérieur de moi, je sens un bien-être absolu [...]. C'est vraiment, j'ai l'impression que je suis dans une autre dimension. Il n'y a plus de limitation. Il n'y a plus rien. Il n'y a plus de doute, il n'y a plus de peur, il n'y a plus d'angoisse, c'est vraiment un état d'extase intérieurement, c'est comme ça que je le sens [...]. C'est grandiose [...]. J'ai peut-être pas les mots, c'est tellement grand [...]. Quand je donne la guérison, en tant qu'instrument je fais le vide complètement. Parce que si je ne fais pas le vide, j’y arrive pas. En fai- 
sant le vide, je comprends que moi je deviens comme un instrument de Lumière, qui apporte de la Lumière à la personne qui a besoin de guérison. À ce moment-là, je sens le bien-être, l'harmonie, la santé, la paix, la purification qui s'installe et tout ça, chez la personne qui a besoin, et moi par le fait même, bien je ressens que j'en reçois des bénédictions, autant moi aussi j'en recueille de la sérénité intérieure, spirituelle, psychologique, émotionnelle, physique aussi.

Sylvie, que nous avons citée plus haut, travaille comme guérisseuse et médium à l'église spirituelle depuis plusieurs années. Née dans une famille catholique non pratiquante, elle est entrée en contact avec le spiritualisme il y a presque trente ans et ne fréquente pas d'autres groupes religieux. Sylvie prie et médite tous les jours: "Je travaille constamment sur mes énergies [...]. Constamment, le soir en méditation, j'entre dans une espèce d'énergie spirituelle, avec mes guides qui est spéciale, puis je travaille toutes les énergies de protection.» Cependant, quand nous lui demandons si elle se dirait «spiritualiste », elle nous répond, d'un air surpris: «Je n'y ai jamais pensé comme ça. » Plusieurs autres membres de l'ÉSG très actifs, réagissent de la même façon, bien que quelques-uns nous disent: «Oui, je suis spiritualiste.» Pour sa part, Nancy précise: "Je dirais plutôt que je suis spirituelle. " En fait, le mot religion est presque absent des récits que nous avons recueillis. Les répondants évoquent leur "voie spirituelle", leur «cheminement spirituel ", leur "pratique spirituelle» et ainsi de suite. Les mots religion et religieux sont rarement prononcés. L'identification à la religion, et plus particulièrement à la religion spiritualiste, ne semble pas refléter le degré de participation aux activités de l'ÉSG, ni l'intensité de la foi, ni la pratique spirituelle quotidienne. Par contre, nos interviewés expriment le sentiment d'être « chez soi » dans l'ÉSG, et surtout dans les groupes et activités dirigés par Michel, où ils trouvent "quelque chose qui me ressemble» (Blaise).

\section{Conclusion}

La mondialisation a occasionné de nouvelles réflexions en anthropologie sur les affiliations nationales (Alonso 1994), ethniques (Fischer 1986), mais aussi religieuses. Les chercheurs constatent notamment que les identités exclusives et primordiales cèdent la place à de nouvelles identités religieuses (Meintel et LeBlanc 2003) à travers la conversion (Mary 2001) ou la reconversion (LeBlanc 2003), sinon par le cumul d'identités religieuses (Meintel 2003). Alors que l'espace virtuel et les mobilités de toutes sortes piquent au vif l'intérêt des chercheurs, plusieurs auteurs insistent sur l'importance du 
milieu «local », soit du milieu plus proche, plus intime, celui des relations face à face, du sensoriel et du corporel (par exemple, Hannerz 1996; Appadurai 1996). Que ce «niveau local» soit centré sur un seul locus physique ou qu'il prenne la forme d'un réseau délocalisé, il représente, selon Appadurai, " a structure of feeling, a property of social life and an ideology of situated community» $(1996,189)$. Dans cette même optique, on réfléchit sur la notion du «chez-soi », du home (Olwig 1997). En effet, il ne s'agit plus du village (imaginé) étanche et fermé d'autrefois, mais bien d'un nexus de relations et significations. Hoffman parle même d'un deuxième chez-soi, le home de la vie adulte, "où idéalement la force de notre premier patrimoine peut être transposée ou mise en dialogue avec nos expériences ultérieures » (1999, 62 , notre traduction).

L'Église spirituelle de la guérison offre un chez-soi religieux à ses membres et, par son absence de dogmatisme, permet à ces derniers de conserver leurs référents et croyances religieux antérieurs, tout en renforçant une attitude de foi. Pour nos interviewés qui fréquentent l'église depuis quelques années, il ne s'agit pas simplement d'un refuge, mais d'une sorte de foyer qui nourrit et fait grandir la foi déjà présente. Nous avons signalé la pertinence des pratiques de guérison et de médiumnité qui servent à rehausser la foi, parallèlement à l'expérience sensorielle et interpersonnelle. Nous tenons à rappeler que nous parlons ici d'une communauté, et non d'une vie sociale communautaire telle qu'elle peut s'élaborer au sein de religions plus sectaires, dans le sens wébérien du terme, soit de prôner une rupture avec le monde ambiant (Hervieu-Léger 1999, 191 ; 2001, 71). Parfois, les individus se fréquentent à l'extérieur des activités religieuses sur une base amicale, mais normalement il s'agit de rencontres dyadiques qui ne concernent pas d'autres membres de l'église. Comme nous l'avons précisé plus haut, non seulement aucune activité n'est prévue pour les enfants, mais en outre cette église ne propose pas d'activités de sociabilité (soupers bénéfices, clubs de femmes ou de jeunes). Néanmoins, une forme de communauté se crée, particulièrement dans les groupes fermés où les mêmes individus se rencontrent régulièrement. Notre observation participante d'un groupe fermé, de la guérison et des cultes hebdomadaires pendant plusieurs années nous a fait comprendre que ce partage pendant une heure ou deux peut sembler plus profond et plus intense que la vie sociale de tous les jours. Les membres de ces groupes ne connaissent pas toujours les noms de famille les uns des autres et, pourtant, ils partagent des expériences qui sont très significatives pour eux et qu'ils ne communiquent pas à leurs proches dans le quotidien. En même 
temps que les croyances des membres sont d'une grande variabilité, ces derniers reconnaissent des convergences dans leurs démarches et expériences spirituelles, comme le décrit Hervieu-Léger par rapport aux communautés religieuses «processuelles» $\left(1999,198^{17}\right)$. Il s'agit en fait d'un communitas (Turner 1975) entre croyants au sein duquel les croyances particulières sont moins importantes que le croire religieux. Ce communitas est éphémère sur le plan temporel, mais ses effets s'étendent aux pratiques quotidiennes. Enfin, c'est une communalité qui est généralement extérieure aux relations et activités de la vie ordinaire, mais qui les inspire.

\section{Références}

Acedo, E. (2000), "Le magnétisme, un lien entre le corps et l'esprit ", dans P. Wallon, dir., Guérir l'âme et le corps. Au-delà des médecines habituelles, Paris, Albin Michel, p. 53-68.

Alonso, A-M. (1994), "The Politics of Time, Space and Substance. State Formation, Nationalism and Ethnicity ", Annual Review of Anthropo$\log y, 23$, p. 379-405.

Appadurai, A. (I996), "The Production of Locality ", dans Modernity at Large: Cultural Dimensions of Globalization, Minneapolis, University of Minnesota, p. 178-204.

Aubrée, M et F. Laplantine (1990), La table, le livre et les esprits: naissance, évolution et actualité du mouvement social spirite entre France et Brésil, Paris, Lattes.

Barbanell, S. (1940), When Your Animal Dies, London, Psychic Press.

Bıввy, R. (1990), "La religion à la carte au Québec: une analyse de tendances », Sociologie et sociétés, 21, p. 133-145.

Bouchard A. (2001), " "Les nouveaux mouvements religieux" et le phénomène des "sectes" " dans J.M. LARouche et G. Ménard, dir., Étude de la religion au Québec. Bilan et prospective, Québec, Presses de l'Université Laval, p. 271-226.

Braude, A.D. (1989), Radical Spirits, Spiritualism and Women's Rights in $19^{\text {th }}$ Century America, Boston, Beacon.

17. «Si communauté il y a, elle a pour vocation non d'attester une homogénéité des croyances postulée par avance, mais de manifester la "convergence" mutuellement reconnue des démarches des membres. » (Hervieu-Léger 1999, 198) 
Cataldi, L. (1993), Emotion, Depth, and Flesh: A Study of Sensitive Space Relictions on Merleau-Ponty's Philosophy of Embodiment, Albany, Suny Press.

Champion, F. (1990), «La nébuleuse mystique-ésotérique: orientations psychoreligieuses des courants mystiques et ésotériques contemporains ", dans F. Champion et D. Hervieu-Léger, dir., De l'émotion en religion:

- Renouveaux et traditions, Paris, Centurion, p. 17-69.

— (1991), «De la religiosité parallèle en France ou la nébuleuse mystiqueésotérique ", Schweizerische Zeitschrift für Soziologie / Revue suisse de sociologie, 3, p. 651-658.

Csordas, T. (2001), Language, Charisma and Creativity: Ritual Life in the Catholic Charismatic Renewal, New York, Palgrave.

Davie, G. (1990), «Believing without Belonging. Is this the Future of Religion in Britain?", Social Compass, 37, p. 456-469.

Favret-SaAda, J. (1977), Les mots, la mort, les sorts, Paris, Gallimard.

Fisher, M.J. (1986), "Ethnicity and the Post-Modern Arts of Memory », dans J. Clifford et G.E. Marcus, dir., Writing Culture: The Poetics and Politics of Ethnography, Berkeley, University of California Press, p. 194-233.

FonseCA, C. (1991), «La religion dans la vie quotidienne d'un groupe populaire brésilien ", Archives de sciences sociales des religions, 73, p. 125139.

Giddens, A. (1990), The Consequences of Modernity, Stanford, Stanford University Press.

Goodman, F. (1972), Speaking in Tongues: A Cross-Cultural Study of Glossolalia, Chicago / Londres, University of Chicago Press.

- (1988), "Spiritualism», dans How about Demons, Bloomington, Indiana University Press, p. 25-41.

Hannerz, U. (1996), "Transnational Connections ", dans The Local and the Global: Continuity and Change, Londres / New York, Routledge, p. 17-29.

HERVIEU-LEGER, D. (1996), «La religion des Européens : modernité, religion, sécularisation ", dans G. DAvie et D. Hervieu-LéGer, dir., Identités religieuses en Europe, Paris, Découverte, p. 9-23.

- (1999), Le pèlerin et le converti. La religion en mouvement, Paris, Flammarion.

- (200I), La religion en miettes ou la question des sectes, Paris, CalmannLévy. 
Hoffman, E. (I999), "The New Nomads », dans A. Aciman, dir., Letters of Transit: Reflections on Exiles, Identity, Language and Lose, New York, The New Press, p. 35-63.

Laplantine, F., dir. (1985), Un voyant dans la ville. Le cabinet de consultation d'un voyant contemporain, Georges de Bellerive, Paris, Payot.

Leblanc, M-N. (2003), "Les trajectoires de conversion et de l'identité sociale chez les jeunes dans le contexte post-colonial Ouest-Africain : les jeunes musulmans et les jeunes chrétiens en Côte-d'Ivoire ", Anthropologie et sociétés, 27, p. 85-110.

Lemieux, R. (1992a), "Les croyances: nébuleuse ou univers organisé ? ", dans R. Lemieux et M. Milot, dir., Les croyances des Québécois. Esquisses pour une approche empirique, Québec, Université Laval (Les Cahiers de recherches en sciences de la religion 11), p. 26-50.

— (1992b), "Histoires de vie et postmodernité religieuse ", dans R. Lemieux et M. Мıьот dir., Les croyances des Québécois. Esquisses pour une approche empirique, Québec, Université Laval (Les Cahiers de recherches en sciences de la religion 11), p. 187-234.

Lemieux, R. et M. Milot (1992), Les croyances des Québécois. Esquisses pour une approche empirique, Québec, Université Laval (Les Cahiers de recherches en sciences de la religion 11).

Linteau, P.-A., R. Durocher, J.-C. Robert et F. Ricard (I989), Histoire du Québec contemporain, t. II : Le Québec depuis 1930, Montréal, Boréal.

Mary, A. (2001), "Globalisation des pentecôtismes et hybridité du Christianisme africain », dans J.-P. BASTIAN, F. CHAMPION et K. Rousselet, dir., La globalisation du religieux, Paris, L'Harmattan, p. 153-170.

Meintel, D. (2000), "Transnationalité et le renouveau de la vie festive capverdéenne-américaine ", Revue européenne de la migration internationale, $16 / 2$, p. 77-90.

- (2003), «La stabilité dans le flou. Parcours religieux et identités de spiritualistes ", Anthropologie et sociétés, 27/1, p. 35-64.

- (sous presse - a), "When the Extraordinary Hits Home: Experiencing Spiritualism ", dans J.-G. Goulet et B. Miller, dir., Transformative Moments in the Field, Lincoln, University of Nebraska Press.

- (sous presse $-b$ ), «Evénements et non-événements. Une recherche en milieu spiritualiste », dans J. LÉvy et J.-I. Olazabal, dir., Anthropologie et événement, Ste-Foy, Presses de l’Université Laval.

Meintel, D. et M.-N. LeBlanc (2003), "La mobilité du religieux à l'ère de la globalisation ", Anthropologie et sociétés, 27/1, p. 5-11. 
Milot, M. (1992), "Typologie de l'organisation des systèmes de croyances », dans R. Lemieux et M. Milot, dir., Les croyances des Québécois. Esquisses pour une approche empirique, Québec, Université Laval (Les Cahiers de recherches en sciences de la religion 11), p. 115-133.

Olwig, K.F. (1997), "Cultural Sites : Sustaining a Home in a Deterritorialized World », dans K.F. Olwig et K. Hastrup, dir., Siting Culture: The Shifting Anthropological Object, New York / Londres, Routledge, p. 17-38.

Polo, A.-L. (2001), Appropriation de l'espace et pratiques municipales de gestion de la diversité ethnoculturelle. Le cas des lieux de culte pentecôtiste, Rapport de recherche, Montréal, INRS-Urbanisation, culture et société.

Pouillon, J. (1993), Le cru et le su, Paris, Seuil.

SAmarin, W. (1972), Tongues of Men and Angels: The Religious Language of Pentecostalism, New York, Macmillan.

Turner, V. (1975), Revelation and Divination Ithaca, New York, Cornell University Press.

Welte, B. (1984), Qu'est-ce que croire? / trad. par M. Thoma et J.-C. Petit, Montréal, Fides, p. 60.

Zaretsky, I. (1974), «In the Beginning Was the Word: The Relationship of Language to Social Organization in Spiritualist Churches », dans I. ZARETSKY et M.P. Leone, dir., Religious Movements in Contemporary America, Princeton, Princeton University Press, p. 166-222.

\section{Résumé}

À première vue, l'objet de notre enquête, soit les croyances des spiritualistes, peut sembler très flou. Nous présenterons tout d'abord une topologie heuristique des croyances de spiritualistes membres du groupe sur lequel est centrée notre enquête. Le spiritualisme se distingue en effet par certaines croyances - on peut contacter les esprits des défunts et ces derniers peuvent venir en aide aux vivants - ainsi que par sa conception des dons spirituels dont chaque être humain serait doté. La guérison par les mains ou à distance, la clairvoyance, la clairaudience et la psychométrie (voyance à travers les objets) figurent ainsi parmi les dons possibles. Puis, nous essaierons de montrer que le caractère apparemment flou des croyances (la diversité de leur provenance, la variabilité des croyances au sein du groupe spiritualiste) n'empêche pas que la croyance religieuse s'épanouisse dans la vie de ces gens. Nous examinerons à cet effet de quelle manière les pratiques spécifiques au spiritualisme (par comparaison aux religions classiques en Occident), soit la 
médiumnité (voyance) et la guérison, servent à renforcer la foi. Finalement, nous nous pencherons sur la question de l'appartenance religieuse de ces personnes (souvent, elles ne se considèrent pas comme "spiritualistes") et sur le rôle de l'église qui fait l'objet de notre enquête (nommée l’Église spirituelle de la guérison) dans la foi religieuse de ses membres.

\begin{abstract}
At first, the object of our study, spiritualists religious beliefs, may seem quite amorphous. I will first present a heuristic topology of the beliefs of the spiritualists who are members of the group that is the focus of my study. Spiritualism is distinct from many other groups present in North America by virtue of certain beliefs-e.g., that one can contact the spirits of the dead and they in turn can help the living-as well as in its notion of the spritual gifts with which every human being is endowed. Healing by the laying on of hands or at a distance, clairvoyance, clairaudience and psychometry (clairvoyance via material objects) are among the possible gifts one may have. Then I will try to show that the apparent vagueness of spiritualist beliefs (their varied provenance, the variablity of beliefs among members of the group) does not hinder the development of faith among these individuals. Specific practices such as mediumship (clairvoyance) and healing, I argue, help reinforce faith. Finally, the question of the religious belonging of the members of the group is raised (often they do not identify as "spiritualists"). Here we look at the role of the church we studied (called the Spiritual Church of Healing) as regards the faith of its members.
\end{abstract}

